\title{
Las principales celebrities en Twitter: análisis de su comunicación e influencia en la red social
}

\section{As principais celebridades no Twitter: análise de sua comunicação e influência na rede social}

Cristóbal Fernandez Muñoz ${ }^{1}$

Maria Luisa García-Guardia²

Resumen El cambio de paradigma en la capacidad de comunicar que han hecho posible los medios digitales produce una transformación en la forma en que las estrellas del deporte, el cine o la música se relacionan con sus audiencias en cuestión de segundos. ¿Cómo se comunican realmente estas personalidades con sus seguidores en Internet, usando como medio la tecnología de las redes sociales y en concreto la plataforma Twitter? Este estudio seleccionó a las personas que tenían en 2015 el mayor número de seguidores en redes sociales a nivel mundial. Las formas de comunicación de cada una de las personalidades tienen aspectos diferentes y complementarios. Se analizó la temática y el tipo de contenido empleado: texto, imagen, y/o vídeo, utilizado con mayor frecuencia, así como el grado de diálogo alcanzado con los seguidores.

Palabras clave: Redes sociales; Twitter; celebridades; interacción social; fans

Abstract The paradigmatic shift in the ability to communicate, which has been made possible by digital media, produces a transformation in the way sports, film or music stars can relate to their audiences in a matter of seconds. How do these figures really communicate with their followers on the Internet, using social networking technology, specifically Twitter, as a platform? This study selected people with the highest number of followers on social networks worldwide

\footnotetext{
${ }^{1}$ Universidad Complutense de Madrid, Espanha.

E-mail: cristobal.fernandez@ccinf.ucm.es

${ }^{2}$ Universidad Complutense de Madrid, Espanha.

E-mail: mluisag902@gmail.com
} 
in 2015. Forms of communication of each of the personalities have different and complementary aspects. The subject and content type was analyzed -text, image, and/or video were most commonly used-as well as the level of dialogue reached with followers.

Keywords: Social networks; Twitter; celebrities; social interaction; followers 


\section{Introducción}

Un líder de opinión es una persona u organización que tiene la capacidad de ejercer influencia sobre las actitudes o la conducta de otros individuos, hacia ciertas formas de pensar o de actuar, en virtud de que es percibido por los demás como una autoridad o una fuente confiable de información, noticias, o reflexiones. Sin embargo, una celebridad (a veces referido con el anglicismo celebrity) es una persona famosa, ampliamente conocida y reputada que tiene un alto grado de atención por parte del público y de los medios de comunicación. No siempre un líder de opinión es una celebridad y viceversa. Tiene que existir cierto nivel de interés público en la persona, que puede estar motivado o no, con la razón por la que esa persona es famosa. Muchas celebridades lo son sólo durante un período concreto (MARSHALL, 1997).

Los líderes de opinión y las celebridades adquirieron su máximo apogeo con la llegada de los medios de comunicación de masas. Fueron los propios medios los que en muchos casos otorgaron ese status a aquellos que producían identificación con las audiencias por su grado de notoriedad pública -en un círculo de retroalimentación constante-, su mero cargo o posición, sus éxitos y logros, y en menor medida por sus conocimientos, habilidades o ejemplaridad. De cualquier forma, se trataba de individuos influyentes sobre la denominada "opinión pública" gracias a la comunicación de masas.

Con la llegada de los medios sociales, sólo posible primero con la irrupción de la web 2.0 en la primera década del siglo XXI, hemos asistido a la convivencia del modelo tradicional del líder de opinión junto con la nueva revolución de la llamada nueva "democracia" de la comunicación social (KIM, 2009). De repente, ciudadanos anónimos con el poder de generar contenidos y compartirlos masivamente tenían al alcance de su mano la oportunidad de tener un alcance multiplicado, como nunca hasta entonces había ocurrido. Las reglas del juego cambiaron, el control del mensaje, de la comunicación, de las marcas, saltó por los aires para dejar paso a la capacidad de influencia en manos de los individuos, de los públicos, de los consumidores, que por primera 
vez se situaban al nivel de las organizaciones, de los medios y las agencias en cuando a las posibilidades potenciales de generar contenidos y difundirlos masivamente. Potencialmente cualquiera podía convertirse en un influenciador gracias a los nuevos medios al alcance de todos, que evidenciaban que el poder de la comunicación amplificada ya no estaba sólo en manos de quienes habían ostentado su control hasta ese momento.

En este nuevo escenario digital, las redes sociales fueron el mayor ejemplo de las posibilidades que ofrecían las nuevas herramientas de comunicación interpersonal, haciendo saltar por los aires las viejas limitaciones espaciotemporales y de alcance de antaño. En este contexto, las celebridades rápidamente "[...] incorporaron los medios sociales para mantener y reforzar la vinculación con sus seguidores” (MARSHALL, 2010; MARWICK \& BOYD, 2011; LEE \& SHIN, 2012; LEE \& JANG, 2013). Una de las cuestiones que se plantean es si son las propias personalidades las que escriben y envían sus comunicaciones en las redes sociales o si lo hacen a través de un equipo de comunicación y relaciones públicas. Sea como fuere, el llamado carácter de "Celebridad" se ha convertido en una serie de estrategias y prácticas que buscan darle una continuidad a la fama (MAWERICK \& BOYD, 2011), y es frecuente encontrar una combinación de ambos planteamientos personales y corporativos, pero deseablemente respondiendo a una estrategia ordenada.

Con la globalización de Internet, comenzaron a aparecer primero las webs oficiales de celebridades. En ellas se mostraba información estática sobre su biografía, actividad profesional, giras, prensa... Sin embargo, este medio no permitía la interacción celebridad-fan. Todo cambió cuando en 2004 apareció Facebook, y en 2006, lo hizo Twitter. Pronto las celebridades abrieron páginas oficiales de Facebook y empezaron a "tuitear", término utilizado al hecho de crear y publicar mensajes en la red social Twitter. Los fans comenzaron a seguirlos en las redes sociales y se posibilitó la comunicación directa entre celebridades y sus fans, ahora convertidos en seguidores de sus redes sociales. 
Mientras que la imagen pública tradicional sigue vigente, las redes sociales permiten en teoría una relación más cercana entre la celebridad y sus seguidores, o es el tipo de relación que perciben muchos seguidores. Las actualizaciones de estado o publicaciones en las redes sociales tratan de mostrar su lado más humano, compartir información de cualquier tipo, vincularse con otras celebridades y, en definitiva, comunicar un hecho desde su punto de vista personal. De modo que la personalidad publica su estado y los seguidores comentan, comparten y/o "les gusta” dicha publicación. Y por encima de toda publicación está la tarea de crear, respaldar y modificar la imagen pública de la celebridad.

Un fenómeno fruto de esta repercusión de las redes en todos los ámbitos de la sociedad es el de los nuevos famosos que surgen desde las mismas redes sociales, sin haber tenido antes una presencia destacada en los medios tradicionales. A esto se le denomina micro-celebridad, e implica una consideración de los amigos y seguidores en las redes como base de fans, buscando la popularidad como una meta, y gestionando esta base de fans mediante técnicas de afiliación efectivas, asemejándose bastante a aquellas técnicas usadas por personalidades famosas para manejar sus audiencias por Facebook o Twitter.

La competencia se multiplica. En palabras de Gallagher (2015), ya no es aceptable que las celebridades tengan un papel pasivo en su imagen, ni en el uso y distribución de la misma. Sino que ahora deben difundir sus actividades diarias, mostrando a sus fans lo que sucede en su vida más personal. Basta con fijarse en que en las cuentas de redes sociales, parecería que las estrellas de cine pasan más tiempo promocionando una película que trabajando en ella, para comprobar que ahora es fundamental la autopromoción si se quiere mantener el status de celebridad. La fama cuesta, y en el escenario digital hay que trabajarla diariamente y ser muy interactivo, para no pasar desapercibido en un escenario plagado de nuevos influenciadores anónimos que buscan convertirse en nuevas celebrities.

Actualmente es difícil hacer una separación clara entre medios masivos y medios interpersonales; incluso los investigadores hablan ahora 
de "comunicaciones personales masivas". Twitter es un ejemplo muy destacado de este fenómeno, porque los usuarios con más seguidores son precisamente celebridades; individuos que se comunican directamente con su público a través de sus Tweets, saltando los tradicionales intermediarios de los viejos medios de comunicación masivos (WU y cols., 2011, pp. 705).

Para MARWICK y BOYD (2011), Twitter permitiría transformar la relación parasocial entre fans y celebridades. Como las propias autoras señalan "la práctica de la celebridad [en Twitter] precisa de observar a los seguidores como fans” (2011, p. 144). (CARO CASTAÑO, 2015). La posibilidad de reciprocidad y de apelación directa de los fans a la cuenta de la celebridad sería suficiente para que se pudiese hablar de una experiencia social y no parasocial. Sin embargo este trabajo plantea que se produce el fenómeno justamente contrario: las redes sociales contribuyen a potenciar la idea de una presunta horizontalidad y simetría en la interacción comunicativa entre fans y celebridad que no se da en la realidad, que es además contraria a la propia noción de celebridad, y que contribuye a ocultar realmente las relaciones de poder que subyacen en estos espacios.

Aunque dado que se trata de un fenómeno reciente y no hay aún mucha investigación específica el respecto, los datos de estudios como el realizado por Page (2012, p. 11), reflejan el comportamiento de las cuentas de Twitter de celebridades respecto a usuarios no celebridades y empresas, poniendo de manifiesto la supremacía en las redes sociales de las cuentas de celebridades ya mencionada. Del total de tweets de las celebridades, más del $60 \%$ son destinados a compartir actualizaciones de estado (fotos, lugares, qué hacen, qué piensan, etc.). Mientras que solo un $32 \%$ van dirigidos a otros usuarios. Y un escaso $5 \%$ a retweets. En cambio, los usuarios no celebridades, dirigen más sus tweets a otros usuarios y retweets y comparten menos actualizaciones.

La presente investigación trata de contribuir al análisis de las comunicaciones que realizan las principales celebridades dentro de la plataforma social Twitter, estudiando el grado de interacción a través 
del (RTs) y favoritos (FAVs) y el impacto y la repercusión que eso tiene. Asimismo, investigar el formato de la comunicación de las celebridades en Twitter atendiendo al texto, la imagen y el video. Las hipótesis de trabajo son, por una parte que existe una correlación referida a los fans respecto a sus celebridades entre el número de RTs (veces que los seguidores "re-comparte un mensaje") y el número de FAVs (número de seguidores que marcan como favorito un mensaje), por otra que publican más mensajes en Twitter con contenido visual o audiovisual y que hay una tendencia por parte de las celebridades a interactuar con sus seguidores siendo aquellas que más interactúan en Twitter las seguidas por más personas.

Más recientemente, el estudio realizado por Kim \& Song (2016) investigó cómo la forma en la que se comportan las celebridades en sus medios de comunicación social, y particularmente Twitter, afecta a la percepción que los fans tienen de ellos. Utilizando una muestra de fans en Twitter seguidores de celebridades, los resultados encontrados mostraron que el hecho de compartir no solo su vida laboral sino también personal (por ejemplo, compartiendo su vida con amigos, familia, gustos, aficiones) mejoraba la percepción de los fans sobre ellos. Además, el hecho de compartir tweets de terceros aumentaba el grado de presencia social y se observó que la actividad en redes sociales correlacionaba positivamente con el grado de interacción parasocial de las celebridades.

\section{Metodología}

A la hora de elegir las celebridades representativas para el estudio, se realizó la selección en base a su influencia y presencia en redes sociales, tomando en cuenta las 10 que más seguidores tenían a nivel mundial y con presencia en Twitter. El ámbito donde se realizó la investigación fue la red social Twitter ya que el nivel de igualdad y transparencia es mayor que otras como Facebook y permite investigar de forma homogénea y clara. Las diez celebridades top corresponden a una de estas tres categorías: Deporte, música o cine. 
El análisis de los textos se realizó tomando como fuente los contenidos de las celebridades publicados. El grado de multiplicación de los mensajes que transmiten fue un elemento importante en la investigación, ya que es lo que indicó el grado de alcance de su comunicación. En este sentido se contó con tres elementos de medida que aportan información sobre cuánta repercusión tiene su imagen y lo que transmiten con ella: volver a compartir el contenido publicado por la celebridad o "retuit" (RT), marcarlo como favorito (FAV) o responder al mismo (Reply). La información que suele contener la respuesta puede ser un comentario en forma de texto escrito (a. De un autor que es citado, b. De un autor que no es citado, c. Creado por él, d. Una combinación de los anteriores), de imagen con texto, Fotografía (la foto que es compartida o comunicada puede ser a su vez de a. De un autor al que se le da crédito, b. De un autor al que no se le da crédito o porque se desconoce cuál es o porque no se lo quiere dar, c. La fotografía es obra del usuario o fan y lo comparte y el autor no sale en la foto; o d. Cuando el propio autor forma parte de la imagen), o un vídeo (de una fuente externa o del mismo usuario que transmite hechos, información u opinión de una forma audiovisual).

El análisis documental se realizó en febrero de 2015 considerando como técnicas de análisis estadístico la medición de frecuencias, se usaron tablas de contingencia (crosstabs) y se emplearon elementos estadísticos descriptivos. Se implementó la prueba de Kolmogorov Smirnov que indica si los datos siguen una distribución normal o no, así como la prueba de Wilcoxon que compara el número de casos con RTs superiores a FAVs y el número de casos con FAVs superiores a RTs.

\section{Resultados}

En cuanto el nivel de actividad en la red social, las celebridades que más uso realizaron de las redes fueron los cantantes Justin Bieber y Taylor Swift en ese orden, siendo especialmente prolíficos: al primero es a quien le corresponden el 24,7\% de todos los mensajes emitidos analizados y el 21,8\% a Swift. La siguiente celebridad más activa fue la cantante 
Rihanna con un $14,7 \%$ de los mensajes. Tras ella se encontraban con el mismo nivel de comunicación el futbolista Cristiano Ronaldo y la cantante Katy Perry, ambos con un 14,1\%. Después le siguieron los cantantes Shakira con un 10\% y Eminen con el 0,6\%.

El análisis indicó, como se puede observar en la tabla 1, que no se establece una correlación directa entre RT y FAV. Las variables que tienen un carácter cuantitativo presentan una dispersión de los datos. En el análisis de los datos se deduce que no se sigue una distribución normal con respecto al número de retweets y el número de favoritos.

\begin{tabular}{lccccc}
\hline & N & Mínimo & Máximo & Media & $\begin{array}{c}\text { Desviación } \\
\text { estándar }\end{array}$ \\
\hline $\begin{array}{l}\text { Número de retweets que ha tenido } \\
\text { el tweet }\end{array}$ & 170 & 297 & 135588 & 16976,37 & 20767,311 \\
\hline $\begin{array}{l}\text { Número de favoritos que ha tenido } \\
\text { el tweet }\end{array}$ & 170 & 647 & 147285 & 25830,21 & 25710,538 \\
\hline N válido (por lista) & 170 & & & & \\
\hline
\end{tabular}

Tabla 1. Estadísticos descriptivos sobre el impacto de los mensajes con los retweets.

Fonte: Os autores

En cuanto a las temáticas utilizadas en el contenido de los mensajes que utilizan se observa que las que tienen una mayor frecuencia de utilización son las relacionadas con Promoción y Música, con un 19,4\%y 18,2\% respectivamente. Le siguen las temáticas Grammy y Cumpleaños ambas con un 15,3\%de utilización. Las siguientes temáticas más utilizadas en los textos enviados son la clasificada como temas cotidianos con un $8,8 \%$ y la siguiente la temática correspondiente a la familia, con un $6,5 \%$.

En cuanto al idioma que utilizan las celebridades más seguidas en Twitter es el inglés en el 87,6\% de los casos. En castellano se emiten el $10,6 \%$ de los mensajes, principalmente por la comunicación de la cantante Shakira. En su caso se encuentra un aspecto diferenciador de los demás, y es que ella comunica los mensajes en los dos idiomas, en inglés y en castellano para llegar a sus seguidores. En el 1,2\% los 
mensajes emitidos corresponden al idioma portugués, principalmente por los mensajes del deportista Cristiano Ronaldo. Y por último la combinación de castellano-inglés en el mismo mensaje se produce con 0,6\% de frecuencia. En este análisis, conviene diferenciar que los mensajes que comparte Shakira en ambos idiomas los hace por separado, es decir, normalmente no pone en un mismo mensaje el texto en los dos idiomas, sino que emite dos mensajes distintos uno en inglés y el otro en castellano.

De los mensajes publicados, el $54,7 \%$ de ellos no llevan asociada ninguna imagen, publican simplemente texto. En el 45,3\% restante sí que las celebridades prefieren añadir un contenido visual en forma de imagen a sus mensajes en Twitter, por lo que se observa que hay casi una paridad en este análisis.

En el análisis de la integración de la red social Twitter con la red social Instagram para replicar el contenido de los mensajes de una red en otra, en la mayoría de los casos, en el $85,9 \%$ las celebridades no tienen realizada tal integración. Tan sólo en el 14,1\% de los casos han decidido integrar ambas redes y duplicar el contenido de una en la otra cuando hacen sus publicaciones. Así pues se observó un porcentaje bajo de celebridades que publican mensajes en Twitter deforma enlazada a la publicación en Instagram.

En el caso de los mensajes que llevan asociado no imágenes sino vídeos, se observó que el 92,2\% de los mensajes publicados no llevan asociado ningún contenido audiovisual. Tan solo en el 7,1\% de los casos prefieren añadir el uso del vídeo adjunto a sus mensajes, por lo que se observa un bajo uso del vídeo en la comunicación vía Twitter.

\section{Conclusiones}

En el contexto de las diez personas más seguidas en el mundo en las redes sociales, dentro de la red social Twitter las personalidades Justin Bieber y Taylor Swift fueron los más activos en su comunicación con los seguidores. Por otro lado, la lista de este top 10 la cerraban los que fueron menos activos en 2015, Eminem y Shakira. 
Los resultados permitieron rechazar la hipótesis de que existe una correlación entre el número de retweets -RTs- (veces que los seguidores "recomparte un mensaje") y favoritos -FAVs- (número de seguidores que marcan como favorito un mensaje) que reciben las celebridades por parte de sus fans. De hecho después del análisis se observó que los datos no cuentan con una distribución normal en cuanto al número de veces que un mensaje de una celebridad es "re-compartido" o RTs con el número de veces que los usuarios han marcado el mensaje como favorito o FAV.

Sí que se pudo confirmar la hipótesis de que los seguidores toman más la acción de considerar un mensaje de una celebridad como favorito (FAV) que la acción de "re-compartir" con todos sus seguidores. Se comprobó que hay mayor cantidad de personas que marcan como favorito un contenido al de personas que lo comparten con sus seguidores a través del "re-compartir" o RT. Lo que significa que toman la opción más "segura" en el sentido de que no hacen tan público y visible sus gustos y apoyo a una celebridad que puede o no ser del agrado de sus propios seguidores.

Se rechazó la hipótesis que consideraba que hay una mayor publicación de mensajes en Twitter que adjuntan imágenes que mensajes que no las llevan. El estudio permitió observar claramente que el número de mensajes que se publican con fotos es menor a los que publican sin fotos. Es decir su publicación se hace con mucha frecuencia, un 45,3\% de las comunicaciones que se realizan, pero no llega a ser la mayoría. Sí se pudo confirmar sin embargo el mayor uso de fotos que de vídeos, que se había considerado igualmente como hipótesis.

Se rechazó la hipótesis de que hay una tendencia por parte de las celebridades a interactuar con sus seguidores en respuesta a los mensajes que publican en la red social Twitter. La mayoría de las celebridades prácticamente no interactúan con sus seguidores cuando estos responden o comentan a sus mensajes. Solo ocurrió en el caso de Justin Bieber. En el mismo sentido se rechazó la hipótesis de que las celebridades que más interactúan en Twitter con sus seguidores son los que son seguidos por más personas. 
Se confirmó que los temas de música y promoción son los preferidos por las celebridades en los mensajes que envían por Twitter. Para Katy Perry la temática más importante es la promoción, para Shakira la familia, para Eminem y Rihanna la música, para Justin Bieber la interacción, para Taylor Swift los grammys.

Así pues, por lo que al uso de Twitter se refiere, hay que derribar algunos mitos sobre el uso de los mayores influenciadores, al menos en cuanto al número de seguidores se refiere. Ni son tan interactivos, ni son tan creativos como podría pensarse.

El presente trabajo no deja de ser una contribución a un ámbito que decididamente debe seguir siendo investigado y que próximas investigaciones contribuirán a ampliar en su conocimiento desde una perspectiva profesional y académica.

De cualquier forma, la capacidad de influencia, ya sea de personas naturales o jurídicas, anónimas o no, va a depender, de nuevo en el nuevo escenario digital, de la confianza, la autenticidad, el entendimiento mutuo y la capacidad para aportar y recibir valor de los demás, desde la responsabilidad social y la ética, utilizando acciones comunicativas desde una posición bidireccional y simétrica con dichos públicos, donde el diálogo cobre un nuevo protagonismo, donde es posible tener un momento de gloria fugaz en el universo online pero donde es necesario construir una relación estable para realmente ser un influenciador sólido y con posibilidades de éxito en el medio y largo plazo en el nuevo escenario digital.

\section{Referencias}

CARO CASTAÑO, L. Relaciones e interacciones parasociales en redes sociales digitales. Una revisión conceptual. Icono, 14, v. 13, p. 23-47, 2015. doi: 10.7195/ri14. v13i2.853.

GALLAGHER, M. John Berger, Paris Hilton, and The Rich Kids of Instagram: The Social and Economic Inequality of Image Sharing and Production of Power 
Through Self-Promotion. 2015. Recuperado de: http://scholarship.claremont.edu/ scripps_theses $/ 545 /$.

KIM, J.; SONG, H. Celebrity's self-disclosure on Twitter and parasocial relationships: A mediating role of social presence. Computers in Human Behavior, v. 62, p. 570-577, 2016.

KIM, Y. Synthesizing Paradigmatic Approaches of the Public Relations Theories: “The Public Relations Democracy" as an Integrating Paradigm. In: ANNUAL MEETING OF THE INTERNATIONAL COMMUNICATION ASSOCIATION, New York, 2009. Proceedings... New York, 2009.

LEE, A. Y.; LABROO, A. A. The effect of conceptual and perceptual fluency on brand evaluation. Journal of Marketing Research, 41 (2), p. 151-165, 2004. Recuperado de: http://journals.ama.org/doi/pdf/10.1509/jmkr.41.2.151.28665.

MARSHALL, P. D. Celebrity and Power: Fame in Contemporary Culture. Minnesota: University of Minnesota Press, 1997.

. The promotion and presentation of the self: celebrity as marker of presentational media. Celebrity Studies, v. 1, n. 1, p. 35-48, 2010. Recuperado de: http://www. tandfonline.com/doi/pdf/10.1080/19392390903519057.

MARWICK, A. E. Status update: Celebrity, publicity and self-branding in Web 2.0. Universidad de New York, 2010 - Tesis doctoral. Disponible en: http://gradworks.umi. com/34/26/3426961.html.

.; boyd, d. To See and Be Seen: Celebrity Practice on Twitter. Convergence: The International Journal of Research into New Media Technologies, 17, p. 139-158, 2011. Recuperado de: http://www.tiara.org/blog/wpcontent/uploads/2011/07/marwick_boyd_ to_see_and_be_seen.pdf.

MOU, Y.; MILLER, M.; FU, H. Evaluating a target on social media: From the selfcategorization perspective. Computers in Human Behavior, 49, p. 451-459, 2015. Recuperado de: https://www.researchgate.net/publication/276107099_Evaluating_a_target_on_social_media_From_the_self-categorization_perspective.

PAGE, R. The linguistics of self-branding and micro-celebrity in Twitter: The role of hashtags. Discourse \& Communication, v. 6, n. 2, p. 181-201, 2012. Recuperado de: https://ra.le.ac.uk/bitstream/2381/13398/1/SelfbrandingandMicrocelebridadinTwitter. pdf.

WU, S.; HOFMAN, J.; MASON, W.; WATTS, D. Who Says What to Whom on Twitter. In: WWW '11: INTERNATIONAL CONFERENCE ON WORLD WIDE WEB, 20, 2011, New York. Proceedings... p. 705-714. ACM New York, NY, USA, 2011. Recuperado de: http://www.wwwconference.org/proceedings/www2011/proceedings/p705.pdf. 


\section{Sobre los autores}

Cristóbal Fernandez Muñoz - Profesor Asociado - Facultad CC Información. Departamento de Comunicación Audiovisual y Publicidad II, Universidad Complutense de Madrid, España.

Maria Luisa García-Guardia - Profesora Contratada Doctora en la Universidad Complutense de Madrid, en la que lleva trabajando desde 2004. Desde el año 2003 es doctora por la UCM con calificación Sobresaliente Cum laude por unanimidad. Es también licenciada en la Facultad de CC. De la Información con la especialidad en Publicidad y Relaciones Públicas. Todos los títulos son de la Universidad Complutense de Madrid.

Data de submissão: 01/11/2016

Data de aceite: 08/12/2016 